\title{
Application of X-Ray Diffractometry in Exploration and Research for Atomic Minerals - Yamuna
}

Singh, Atomic Mineral Directorate for Exploration and Research, DAE, Hyderabad - 500016

On the analogy of the diffraction of light wave by gratings, it was observed that crystalline substances having an orderly arrangement of atoms along particular directions in the crystal lattice act similar to grating whose particular plane at a fixed angle diffracts the X-ray radiation. This happens only when the wavelength is of the order of the repeat interval of atomic planes. Such reflection of X-ray radiation is called diffraction, and the angle at which the planes diffract the beam is known as Bragg's angle. As almost every matter consists of selected groups of atoms bonded to each other in different fashion, the repeat distances and the planes for each would be different. It is this property of the crystalline matter that is used to investigate the nature of the atomic arrangement in a particular matter, compound, and metal. The mineral aggregates which defy characterisation by conventional methods due to various reasons are easily identified by X-ray diffractometry (XRD).

The X-ray commonly used in diffraction work is produced by bombarding a target material with fast-moving electrons which are capable of penetrating deep into the orbitals of the atoms. One of the most valuable roles of X-ray analysis is the ability to distinguish between crystalline and amorphous materials. Every crystalline substance has a unique X-ray powder pattern because its position depends on the size of the unit cell, and the line intensity depends on the type of elements present and their arrangement in the crystal. Thus, as each individual could be identified by characteristic fingerprints, in a similar way, an unknown crystalline solid could be pinpointed by its characteristic diffraction pattern.

A comprehensive understanding of ore mineralogy is essential in constraining ore genesis, exploration, and beneficiation. In this context, XRD finds diversified applications in exploration and research for atomic minerals (EARFAM) which are in great demand for the nuclear energy programmes of India.

Summary of the lecture to be delivered at the monthly meeting of the Geological

Society of India on 27 June 2012. 